\title{
Incidencia y determinantes demográficos de la leucemia linfoide aguda en pacientes con cáncer pediátrico, Antioquia
}

\author{
Incidence and demographic determinants of lymphocytic leukemia in pediatric patients with cancer, Antioquia \\ Carlos Alberto Gómez-Mercado ${ }^{*}$ orcid.org/0000-0003-4123-2812 \\ Angela M. Segura-Cardona' orcid.org/0000-0002-0010-1413 \\ Dubán Enrique Pájaro-Cantillo' orcid.org/0000-0002-3728-8699 \\ Miler Mesa-Largo² orcid.org/0000-0001-5177-6325
}

1 Escuela de Graduados. Universidad CES, Medellín, Colombia.

2 Instituto Colombiano de Medicina Tropical, Medellín, Colombia.

\section{Resumen}

Introducción: En el mundo las leucemias agudas son los tumores más frecuentes en la edad pediátrica, de gran interés por sus implicaciones en el niño y su familia. Objetivo: Identificar la incidencia de leucemia linfoide aguda y su asociación con determinantes demográficos en pacientes con cáncer pediátrico, Antioquia, 2017. Materiales y métodos: Estudio observacional, descriptivo, transversal, retrospectivo con intención analítica, sobre la incidencia de leucemia linfoide aguda (LLA) y su asociación con determinantes demográficos no causales de pacientes con cáncer infantil, en 190 registros del Sistema de Vigilancia en Salud Pública (SIVIGILA). Resultados: Las tasas de incidencia de cáncer infantil y LLA fueron 10 casos y 4 casos por cada 100.000 habitantes $\leq 18$ años respectivamente. Las variables asociadas a LLA son: ser hombre (RPa: 1,02 IC95\%: 0,52 - 2,02), residencia rural (RPa: 1,59 IC95\%: 0,55 - 4,56), afiliación al régimen subsidiado (RPa: 1,41 IC95\%: 0,68 - 2,92), edad $\geq 9$ años (RPa: 0,76 IC95\%: 0,38 - 1,50) y oportunidad diagnóstica confirmatoria $\geq 16$ días (RPa: 0,34 IC95\%: 0,10 - 1,15). Conclusiones: Ser hombre, vivir en zona rural y estar afiliado al régimen subsidiado, está relacionado con la incidencia de leucemia linfoide aguda.

Palabras clave: Niños; cáncer; cáncer pediátrico; leucemia; leucemia linfoide aguda (Fuente: DeCS, Bireme).

\begin{abstract}
Introduction: Acute leukemias are the most frequent pediatric malignancies worldwide that have led to a great interest due to their implications for children and their families. Objective: To identify the incidence of acute lymphocytic leukemia and its association with demographic determinants in pediatric cancer patients from Antioquia (Colombia) in 2017. Materials and methods: An observational, descriptive, cross-sectional, retrospective study was carried out with an analytical approach to identify the incidence of acute lymphocytic leukemia (ALL) and its association with non-causal demographic determinants in patients with pediatric cancer. 190 records from the Public Health Surveillance System (SIVIGILA) were analyzed. Results: The incidence rates of childhood cancer and ALL were 10 and 4 cases per 100,000 inhabitants $\leq 18$ years of age, respectively. The variables associated with ALL are: being male (APR: 1.02 95\% CI: 0.52 - 2.02); living in rural areas (APR: 1.59 95\% CI: 0.55 4.56); being affiliated to the subsidized regime (APR: 1.41 95\% CI: 0.68 - 2.92); being $\geq 9$ years of age (APR: $0.7695 \%$ CI: 0.38 1.50); and having a confirmatory diagnosis after 16 days (APR: $0.3495 \%$ CI: $0.10-1.15$ ). Conclusions: The variables related to acute lymphocytic leukemia are: being a man; living in rural areas; and being affiliated to the subsidized regime.
\end{abstract}

Key words: Children; cancer; pediatric cancer; leukemia; acute lymphoid leukemia (Source: DeCS, Bireme).

\footnotetext{
*Autor de correspondencia

Gómez-Mercado CA

e-mail: klargomez@gmail.com
} 


\section{Introducción}

Los tumores en la edad pediátrica son poco frecuentes, y representan aproximadamente del $1 \%$ al 3\% de los tumores en población general(1), sin embargo, constituye una de las principales causas de muerte en menores de 18 años. Según la Organización Mundial de la Salud: "el cáncer en menores de 15 años representa entre un $0,5 \%$ y un $4,6 \%$ de la carga total de morbilidad por esta causa, con tasas mundiales de incidencia, que oscilan entre 50 y 200 casos por cada millón de niños en las distintas partes del planeta" $(2,3)$.

De acuerdo con la información publicada en Globocan (Global Cancer Observatory) en 2018, los tumores más frecuentes en la edad pediátrica a nivel mundial fueron las leucemias con 78.069 casos nuevos, los tumores del sistema nervioso central con 30.106 casos nuevos, el linfoma no Hodgkin con 22.964 y el linfoma Hodgkin con 13.806. En América Latina y el Caribe 32.386 menores de 18 años fueron diagnosticados con cáncer(4). En Colombia, según el reporte de la situación del cáncer en la población atendida en el Sistema General de Seguridad Social en Salud, liderado por el Fondo Colombiano de Enfermedades de Alto Costo, en 2017 se presentaron 1.754 casos de leucemia linfoide aguda, 477 casos de linfoma no Hodgking, 301 casos de linfoma Hodgking y 190 casos de leucemia mieloide aguda en menores de 18 años(5), cifras que coinciden con los reportes del Registro Poblacional de Cáncer de Cali (RPC)(6), el Instituto Nacional de Cancerología(7) y los datos epidemiológicos reportados por el Ministerio de Salud y Protección Social(8).

El cáncer puede ser clasificado en dos grupos: tumores hemato-linfoides (leucemia y linfoma) y tumores sólidos. Los tumores más frecuentes en la infancia (0-14 años) son las leucemias, seguidas de los tumores del sistema nervioso central y los linfomas (Hodgkin y no Hodgkin). En la adolescencia (15-19 años) son más frecuentes los linfomas, seguidos de los tumores de sistema nervioso central y las leucemias; en este grupo de edad se presenta también mayor incidencia de melanomas malignos, tumores germinales y carcinoma de tiroides, comparado con las personas menores de 15 años ${ }^{(9)}$.

La leucemia se define como una aberrante hiperproliferación de células sanguíneas inmaduras que no forman masas tumorales sólidas, que pueden ser agudas o crónicas(10). La leucemia linfoide aguda
(LLA) es una patología inmunofenotípicamente heterogénea, que representa expansiones clonales de linfoblastos en diferentes estadios de maduración, y sustituyen progresivamente a los componentes normales de la médula ósea (MO), invaden la sangre periférica (SP) e infiltran distintos órganos y tejidos, generando la aparición de síntomas y signos clínicos (infecciones frecuentes, fiebre que no desaparece, debilidad y cansancio constante, dolor óseo, inflamación de los ganglios linfáticos (del cuello, las axilas y la ingle), palidez, sangrado o aparición de hematomas con facilidad, dificultad para respirar, dolor de cabeza y hígado o bazo agrandados(11,12).

Para el estudio de las células leucémicas se utilizan técnicas morfológicas, citoquímicas, citogenéticas, de biología molecular e inmunológicas(3), que permiten identificar la línea específica de origen, su nivel de maduración y en algunos casos la clonalidad del proceso. Esto es importante para el entendimiento de las características fenotípicas de las células leucémicas en el diagnóstico de las variedades de la LLA, y la comprensión de las diferentes formas clínicas de expresión de la enfermedad, definir el tratamiento específico y predecir el pronóstico, la evolución y la sobrevida de los enfermos(13).

El pronóstico de la enfermedad depende del tipo del tumor, la extensión de la enfermedad en el momento del diagnóstico y la eficacia del tratamiento. En países desarrollados se estima que aproximadamente entre el $60 \%$ y el $80 \%$ de los niños con cáncer se curan, mientras que en países menos desarrollados, el $50 \%$ de los niños con diagnóstico de cáncer fallece; debido a que se presentan dificultades en el acceso a los servicios de salud, en la oportunidad del diagnóstico e inicio del tratamiento y posiblemente al abandono del mismo por barreras de acceso de tipo económico o geográfico(14,15).

La enfermedad mejora cuando se diagnostica y se empieza a tratar en estadios iniciales, esto permite: usar tratamientos menos intensos, reducir el riesgo de complicaciones relacionadas con la enfermedad y con el tratamiento, disminuir las secuelas orgánicas y psicológicas, los tiempos y costos de hospitalización o servicios tendientes a la atención integral del cáncer y por ende la mortalidad(13).

En la actualidad, el $70 \%$ de los niños con leucemia linfoblástica aguda, tienen una tasa de sobrevida superior a 5 años; sin embargo, la situación es 
diferente para los niños con cáncer de países en desarrollo, donde se estima una sobrevivencia entre $10 \%$ y $20 \%$ menor que la de aquellos en su misma situación en países desarrollados. Las causas de esta situación se adjudican al diagnóstico tardío, el limitado acceso al tratamiento, su abandono y la recurrencia de la enfermedad(13,14).

A diferencia del cáncer en adultos, no es posible implementar estrategias de prevención primaria para el cáncer en niños y adolescentes, así como tampoco existen métodos de tamización que permitan la detección temprana. El cáncer pediátrico se caracteriza por tener una presentación predominantemente tisular, los casos son detectados generalmente de forma accidental, dado que los signos y síntomas son tan comunes que pueden confundirse con otras enfermedades; sin embargo, los tumores diagnosticados en la infancia tienen mejor pronóstico, responden y toleran mejor el tratamiento y se logra una mayor supervivencia comparados con los tumores en adultos(13).

Dado el interés actual del tema, por su impacto en la salud pública y sus implicaciones en todas las esferas económica política y social, este estudio tiene como objetivo identificar la incidencia de leucemia linfoide aguda (LLA) en los pacientes con cáncer infantil en el departamento de Antioquia en el primer semestre del 2017 y explorar la relación con factores demográficos.

\section{Materiales y métodos}

Estudio observacional, descriptivo, transversal, retrospectivo con intención analítica, para la identificación de la incidencia de la leucemia linfoide aguda y su asociación con determinantes demográficos no causales, de pacientes con cáncer infantil del departamento de Antioquia en el 2017.

El proceso de obtención de la información inició con la solicitud formal a la Dirección Seccional de Salud y Protección Social de Antioquia (DSSYPSA) para acceder a los datos de pacientes pediátricos con cáncer en el departamento durante el 2017. Se tomó información de fuente secundaria, a partir del Sistema de Vigilancia en Salud Pública (SIVIGILA) 2017 para el evento de vigilancia en salud pública de cáncer infantil (ficha 115). Se realizó censo de todos los registros y como medida para controlar los sesgos de información, se excluyeron aquellos que tenían datos faltantes por encima del 10\%. En total se incluyeron 190 registros para el estudio.

La variable dependiente del estudio fue el cáncer pediátrico, donde la categoría de riesgo fue la LLA y los demás tipos de cánceres fueron la categoría de referencia. Se incluyó la oportunidad en la confirmación del diagnóstico de los pacientes de cáncer infantil, medida como el tiempo que transcurre desde la fecha de resultado de la prueba diagnóstica que sugiere al médico la probabilidad de cáncer y la fecha de resultado de prueba confirmatoria. El diagnóstico probable se evaluó por signos, síntomas y el diagnóstico confirmatorio por biopsia de médula ósea. Estas variables fueron categorizadas como: oportunidad alta ( $\leq 15$ días) y oportunidad baja o tardía ( $\geq 16$ días). Se consideró otras variables demográficas como: sexo, área de residencia (urbano, rural), edad, municipio de residencia, afiliación al sistema general de seguridad social en salud, hospitalización y condición final del niño.

Se realizó un análisis descriptivo de todas las variables, calculando medidas de frecuencia $y$ medidas de resumen en el software SPSS ${ }^{\circledR}$ versión 21 (licencia Universidad CES). La edad, la oportunidad de diagnóstico probable y confirmatorio fueron recategorizadas y se utilizó la prueba Chi-Cuadrado de Independencia $\left(X^{2}\right)$ de Pearson, con el fin de establecer asociaciones bivariadas entre el cáncer pediátrico, tomando como categoría de riesgo la LLA y las demás variables, donde se hallaron razones de prevalencia crudas, intervalos de confianza y valores $p$. Por último, se realizó análisis multivariado por medio de regresión logística binaria múltiple, utilizando el método introducir, donde se incluyeron variables con valores de $p<0,25$ de acuerdo con el criterio Hosmer y Lemeshow $^{(16)}$ y se calcularon razones de prevalencia ajustadas por las variables independientes, sus intervalos de confianza del 95\% y el nivel de significancia estadística, el cual, se fijó con un valor $p<0,05$.

\section{Consideraciones éticas}

De acuerdo con la Resolución 8430 de 1993 del Ministerio de Salud por la cual se establecen las normas científicas, técnicas y administrativas para la investigación en salud este estudio se clasificó como "sin riesgo" considerando que se trataba de un estudio con información de fuente secundaria y fue avalado por Comité Institucional de Ética en 
Investigación en Seres Humanos de la Universidad CES.

\section{Resultados}

Se analizaron 190 registros de pacientes con cáncer pediátrico, en la figura 1 se presenta la proporción según tipología.

De acuerdo con la distribución de cáncer pediátrico por sexo, en el año 2017, la mayor proporción de casos notificados se presentó en el sexo masculino con el 51,1\%. Se observó que el $85,8 \%$ eran residentes del área urbana, el 59,2\% pertenecían al régimen contributivo en salud, el $77,9 \%$ fueron hospitalizados en algún momento y el 12,6\% fallecieron. Al aplicar la prueba de normalidad de Kolmogorov - Smirnov, para verificar las variables, edad, oportunidad de diagnóstico probable, el valor de $p$ calculado demostró que no se ajustan a una distribución normal. Se observó que el $50 \%$ de los pacientes tuvieron 8 años o menos de edad (RIQ: 9 años), mientras que el $50 \%$ de la población pediátrica tuvo diagnóstico confirmatorio con 1 días o menos (RIQ: 6 días) (Tabla 1).

Geográficamente el departamento de Antioquia se encuentra dividido en 9 subregiones, la más poblada es la del Valle de Aburrá, en la que se encuentra la capital y otros 9 municipios aledaños, representando aproximadamente la mitad de la población de Antioquia; le siguen en número de habitantes las subregiones de Urabá y Oriente. Se observó que la tasa de incidencia de cáncer infantil del departamento en el 2017 fue de 10 casos por cada 100.000 habitantes menores de 18 años, mientras que la tasa de LLA fue de 4 por cada 100.000

Tabla 1. Descripción de los determinantes sociodemográficos de los pacientes con cáncer pediátrico. Antioquia, 2017.

\begin{tabular}{lll}
\hline Variables & n & \% \\
\hline Sexo & & \\
Masculino & 97 & 51,1 \\
Femenino & 93 & 48,9 \\
Área de residencia & & \\
Urbana & 163 & 85,8 \\
Rural & 27 & 14,2 \\
Régimen en salud & & \\
Contributivo & 109 & 59,2 \\
Subsidiado & 72 & 39,1 \\
Especial & 1 & 1,6 \\
Hospitalización & & \\
Si & 148 & 77,9 \\
No & 42 & 22,1 \\
Condición final & & \\
Vivo & 166 & 87,4 \\
Muerto & 24 & 12,6 \\
Edad & $8,0^{*}$ & $9,0^{+}$ \\
Oportunidad de diagnóstico probable & $1,0^{*}$ & $3,0^{+}$ \\
Oportunidad de diagnóstico confirmatorio & $1,0^{*}$ & $6,0^{+}$ \\
\hline *Mediana, +Rango intercuartil (RIQ) & &
\end{tabular}

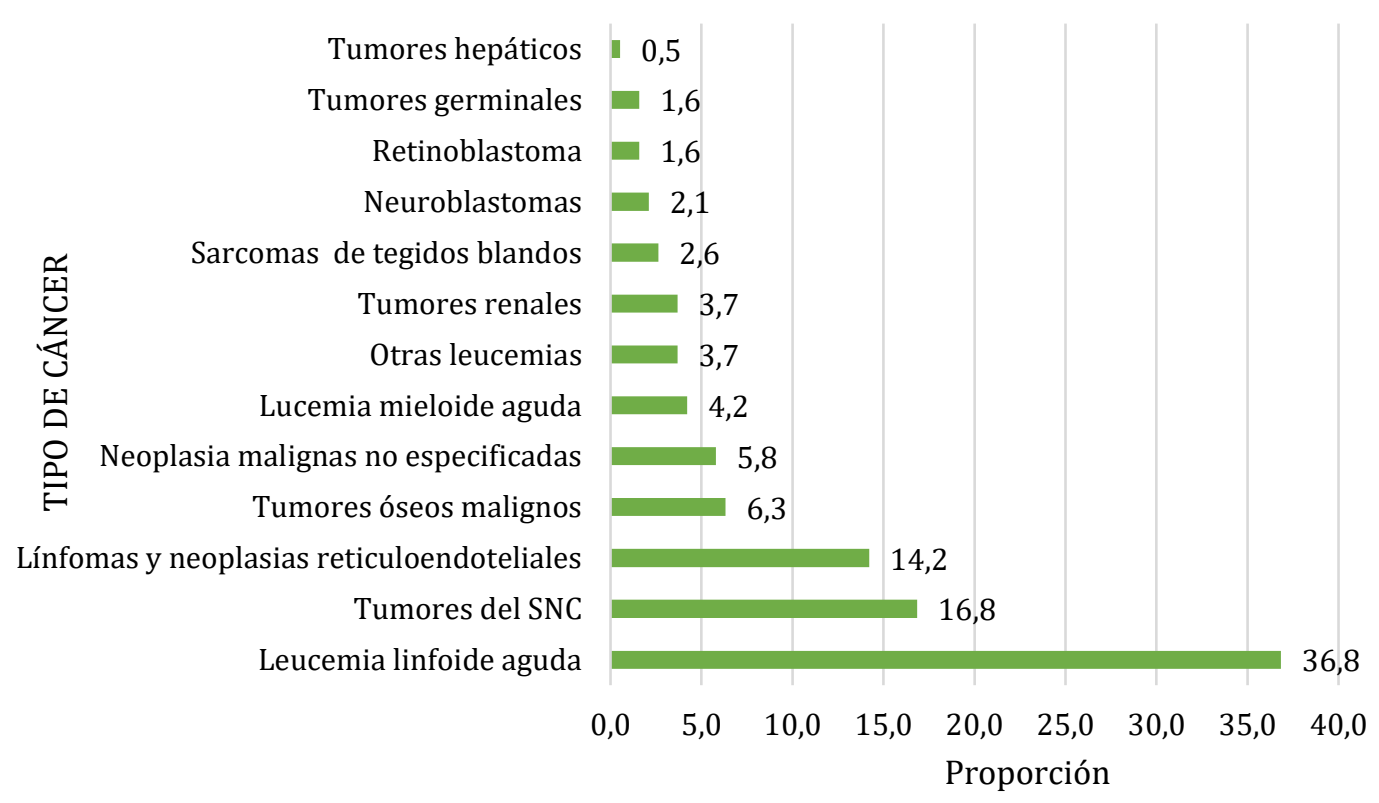

Figura 1. Distribución proporcional del cáncer infantil según tipo, Antioquia, 2017 
habitantes menores de 18. Se destaca que las subregiones del Magdalena medio y el Valle de Aburrá presentaron una tasa de incidencia mayor a la del departamento, 12,7 y 12,1, respectivamente. Las subregiones de Bajo Cauca $(9,1)$, Nordeste $(7,5)$, Norte $(7,4)$, Urabá $(6,1)$ y Suroeste $(5,6)$, reportaron tasas de incidencia por debajo de la media departamental que fue 9,9 .

Se observó que la probabilidad de LLA aumenta en un $1 \%$ en los hombres, al compararlos con las mujeres (RPc: 1,01 IC95\%: 0,56 - 1,84), pero esta asociación no es estadísticamente significativa. La proporción de LLA de los que residían en área rural fue del $18,6 \%$ y tuvieron 1,55 veces mayor probabilidad de tener LLA con respecto a los que vivían en zona urbana. Por su parte, la pertenencia al régimen subsidiado incrementa la probabilidad de aparición de este tipo de cáncer pediátrico en un $25 \%$, al comparar con los de régimen contributivo, sin embargo, esta relación no es significativa.

Con relación a la edad del paciente se evidenció que tener 10 años o más aumenta la probabilidad de LLA
(RPc: 1,40 IC95\%: 0,81 - 2,66). Por su parte, se encontró una asociación significativa $(p=0,020)$ entre tener un diagnóstico probable entre 16 o más días y la disminución de la probabilidad de aparición de LLA (RPc: 0,30 IC95\%: 0,03 - 0,76), al igual que tener un diagnóstico confirmatorio entre 16 o más días, pero esta última asociación no fue significativa (Tabla 2).

Para realizar el análisis multivariado, se incluyó la variable oportunidad en diagnóstico probable, por criterio estadístico de $p<0,05$, también se ingresaron las variables área de residencia (rural), edad $(\geq 9$ años) y oportunidad en diagnóstico confirmatorio ( $\geq$ 16 días) por criterio de Hosmer y Lemeshow, adicionalmente se incluyó el sexo por criterio del investigador. Al realizar el ajuste de la Razón de Prevalencia (RP) mediante el modelo de regresión logística binaria múltiple, tres variables conservaron la condición de aumentar la probabilidad de LLA: ser hombre, residir en el área rural y pertenecer al régimen subsidiado. Sin embargo, ninguna fue estadísticamente significativa (Tabla 3).

Tabla 2. Determinantes sociodemográficos asociados a la leucemia linfoide aguda en pacientes pediátricos

\begin{tabular}{|c|c|c|c|c|c|c|}
\hline \multirow{3}{*}{ Variable } & \multicolumn{4}{|c|}{ Leucemia linfoide aguda } & \multirow{3}{*}{$\begin{array}{c}\text { Razón de Prevalencia (RP) } \\
\text { crudo } \\
\text { [IC 95\%] }\end{array}$} & \multirow{3}{*}{ Valor $p$} \\
\hline & \multicolumn{2}{|c|}{$\mathbf{S i}$} & \multicolumn{2}{|c|}{ No } & & \\
\hline & $\mathbf{n}$ & $\%$ & $\mathbf{n}$ & $\%$ & & \\
\hline \multicolumn{7}{|l|}{ Sexo } \\
\hline Hombre & 36 & 51,4 & 61 & 50,8 & $1,01[0,56-1,84]$ & 0,937 \\
\hline Mujer & 34 & 48,6 & 59 & 49,2 & 1 & \\
\hline \multicolumn{7}{|l|}{ Área de residencia } \\
\hline Urbana & 57 & 81,4 & 106 & 88,3 & 1 & \\
\hline Rural & 13 & 18,6 & 14 & 11,7 & $1,55[0,76-3,92]$ & 0,192 \\
\hline \multicolumn{7}{|l|}{ Afiliación } \\
\hline Contributivo & 39 & 56,5 & 73 & 63,5 & 1 & \\
\hline Subsidiado & 30 & 43,5 & 42 & 36,5 & $1,25[0,72-2,45]$ & 0,350 \\
\hline \multicolumn{7}{|l|}{ Edad } \\
\hline$\leq$ 8años & 40 & 57,1 & 57 & 47,5 & 1 & \\
\hline$\geq 9$ años & 30 & 42,9 & 63 & 52,3 & $1,40[0,81-2,66]$ & 0,200 \\
\hline \multicolumn{7}{|c|}{ Oportunidad en diagnostico probable } \\
\hline$\leq 15$ días & 68 & 97,1 & 92 & 85,2 & 1 & \\
\hline$\geq 16$ días & 2 & 2,9 & 16 & 14,8 & $0,30[0,03-0,76]$ & 0,020 \\
\hline \multicolumn{7}{|c|}{ Oportunidad en diagnostico confirmatorio } \\
\hline$\leq 15$ días & 61 & 93,8 & 96 & 88,1 & 1 & \\
\hline$\geq 16$ días & 4 & 6,2 & 13 & 11,9 & $0,56[0,15-1,55]$ & 0,223 \\
\hline
\end{tabular}

Tabla 3. Determinantes sociodemográficos que explican la leucemia linfoide aguda, pacientes con cáncer pediátrico

\begin{tabular}{lrrrr}
\hline \multicolumn{1}{c}{ Variable } & RP crudo [IC 95\%] & Valor $\boldsymbol{p}$ & \multicolumn{1}{c}{ RP ajustado [IC95\%] } & \multicolumn{1}{c}{ Valor $\boldsymbol{p}$} \\
\hline Sexo (hombre) & $1,01[0,56-1,84]$ & 0,937 & $1,02[0,52-2,02]$ & 0,938 \\
Área de residencia (rural) & $1,55[0,76-3,92]$ & 0,192 & $1,59[0,55-4,56]$ & 0,385 \\
Afiliación en salud (subsidiado) & $1,25[0,72-2,45]$ & 0,350 & $1,41[0,68-2,92]$ & 0,347 \\
Edad ( $\geq$ años) & $1,40[0,81-2,66]$ & 0,200 & $0,76[0,38-1,50]$ & 0,440 \\
Oportunidad en el diagnóstico probable $(\geq 16$ días) & $0,30[0,03-0,76]$ & 0,020 & $\mathrm{NC}$ & $\mathrm{NC}$ \\
Oportunidad en el diagnóstico confirmatorio ( $\geq 16$ días) & $0,56[0,15-1,55]$ & 0,223 & $0,34[0,10-1,15]$ & 0,085 \\
\hline
\end{tabular}

*No-calculado 


\section{Discusión}

De acuerdo con cifras publicadas por la OMS en el 2016, el cáncer en menores de 15 años representa el $4,6 \%$ de la carga total de la morbilidad por esta causa, con tasas generales de incidencia que oscilan entre 50 a 200 casos por cada millón de niños y 90 a 300 por cada millón de adolescentes(2), superiores al reporte encontrado en el departamento de Antioquia para menores de 18 años, con una tasa de incidencia de cáncer infantil de 10 casos por cada 100.000 y de LLA de 4 casos por cada 100.000 habitantes. Actualmente, la proporción de cáncer en niños es del 84\% en los países con Índice Desarrollo Humano (IDH) bajo, al compararlos con los países IDH alto(17).

En Colombia para el 2016, según cifras del Departamento Administrativo Nacional de Estadística (DANE), se presentaron 838 muertes por cáncer por leucemia, tumores del sistema nervioso central, ojo, tumores óseos, articulares y linfomas ${ }^{(5)}$. De acuerdo a las cifras reportadas en el informe de situación de cáncer en la población adulta atendida en el Sistema General de Seguridad Social en Salud de Colombia de la cuenta de alto costo en el 2017, se presentaron 1754 casos nuevos de LLA, de los cuales 70 se reportaron en Antioquia(7). En países de altos ingresos se obtienen mejores resultados de supervivencia, gracias a la mejor oportunidad en el acceso a los avances tecnológicos y de medicamentos, con una tasa de $80 \%$, mientras que en países de bajos ingresos oscila entre $5 \%$ y $60 \%(18,19)$.

En cuanto a los determinantes demográficos asociados a la leucemia linfoide aguda, no se identificó diferencias significativas en relación al sexo con los diferentes tipos de cáncer, a pesar del ligero predominio masculino; estos datos concuerdan con los publicados por Ezzat et al.(20), pero difiere con lo publicado con Steliarova-Foucher et al.(21), quienes realizaron un estudio de registro poblacional, ideado por la Agencia Internacional para la Investigación del Cáncer en colaboración con la Asociación Internacional de Registros de Cáncer, donde recopilaron datos sobre todas las neoplasias malignas y neoplasias no malignas del SNC diagnosticadas antes de los 20 años en poblaciones cubiertas por registros de cáncer de calidad con datos completos para 2001 a 2010.

En este estudio la residencia rural aumentó la probabilidad de LLA, resultados similares a los reportados por Bona et al.(22), que demostraron que los niños estadounidenses con LLA que viven en áreas de alta pobreza y ruralidad tienen un mayor riesgo de recaída temprana en comparación con aquellos que viven en áreas de baja pobreza a pesar del tratamiento uniforme. Al igual que Gupta et al.(23), hallaron que la distancia desde el centro terciario o la residencia rural predijeron significativamente la supervivencia libre de LLA. Pero contrarios a los hallazgos de Erdmann et al.(24) que encontraron que los determinantes socioeconómicos (entre ellos la ruralidad) no afectaron la supervivencia de los niños con LLA de Alemania Occidental.

Al igual que los estudios de Terwilliger y AbdulHay(25), Rytting et al.(26) y Schraw et al.(27), la edad inferior a 9 años se comporta como un factor que se asocia al desarrollo de LLA. Los niños que fueron diagnosticados después de 16 días presentaron una mayor probabilidad de aumentar la progresión de la enfermedad y consecuentemente el riesgo de muerte, este resultado es similar a lo reportado por Vizcaíno et al., quienes concluyen que la demora en la remisión a un especialista ante la sospecha de cáncer y la iniciación tardía o suspensión del tratamiento, pueden significar la diferencia entre la vida y la muerte(28). Así, la oportunidad diagnóstica probable o confirmatoria, es un importante predictor de la LLA(29).

Se debe leer con especial cuidado los resultados, pues el diseño transversal del estudio limita la inferencia causal, por ello, estos son aplicables sólo a la población en la cual se realizó el estudio. El hecho de tener información secundaria no permitió trabajar con otros confusores importantes para comprender los factores no etiológicos de la LLA y sólo se logró trabajar con determinantes demográficos. La principal fortaleza del estudio fue la selección completa de los casos, pues, se trabajó con el censo total de los registros de pacientes pediátricos del departamento en el 2017, situación que controla en mayor medida los sesgos de selección en el estudio.

Los resultados de este estudio refirman la necesidad de generar acciones e intervenciones desde la promoción de la salud y prevención de la enfermedad, enfocadas a controles variables asociadas al riesgo de LLA. Se requiere la participación de la ciudadana en la formulación de políticas públicas, fomento de redes de apoyo 
comunitario, participación en programas de educación.

\section{Conclusión}

Factores demográficos como ser hombre, residir en zona rural, pertenecer a un régimen en salud que limita el acceso a la prestación de los servicios de salud y un diagnóstico tardío, se han asociado con la presencia de LLA en niños con cáncer.

\section{Referencias}

1. Bray F, Ferlay J, Soerjomataram I, Siegel RL, Torre LA, Jemal A. Global cancer statistics 2018: GLOBOCAN estimates of incidence and mortality worldwide for 36 cancers in 185 countries. CA Cancer J Clin. 2018;68(6):394-424. DOI: 10.3322/caac.21492.

2. Pardo C, Cendales R. Incidencia, mortalidad y prevalencia de cáncer en Colombia 2007-2011. 2015. Disponible en: https://www.cancer.gov.co/files/libros/archivos/incidenci a1.pdf.

3. Tovar JR, Gómez GA. Incidencia de cáncer infantil en una ciudad colombiana. Rev Cienc Salud. 2016 Sep 9;14(03):315-29.

http://dx.doi.org/10.12804/revsalud14.03.2016.01.

4. Global Cancer Observatory [Internet]. [citado 27 de enero de 2019]. Disponible en: http://gco.iarc.fr/.

5. Ospina ML, Huertas JA, Montaño JI, Rivillas JC. Observatorio Nacional de Cáncer Colombia. Fac Nac Salud Pública El Escen Para Salud Pública Desde Cienc. 2015;33(2):262-76. Disponible

en: http://www.scielo.org.co/pdf/rfnsp/v33n2/v33n2a13.pdf.

6. Registro Poblacional de Cáncer de Cali [Internet]. [citado 28 de julio de 2019]. Disponible en: http://rpcc.univalle.edu.co/es/.

7. Instituto Nacional de Cancerologia | Por el control del cáncer [Internet]. [citado 28 de julio de 2019]. Disponible en: https://www.cancer.gov.co/.

8. Pardo C, Cendales R, Pardo C, Cendales R. Cancer incidence estimates and mortality for the top five cancer in Colombia, 2007-2011. Colomb Médica. 2018 Mar;49(1):16-22. DOI: https://doi.org/10.25100/cm.v49i1.3596.

9. Kato M, Manabe A. Treatment and biology of pediatric acute lymphoblastic leukemia. Pediatr Int. 2018;60(1):4-12. DOI: 10.1111/ped.13457.

10. An Q, Fan C-H, Xu S-M. Recent perspectives of pediatric leukemia - an update. Eur Rev Med Pharmacol Sci. 2017 Oct;21(4 Suppl):31-6. Disponible en: https://europepmc.org/article/med/29165768.

11. Marsán Suárez V, Cos Padrón Y, Sánchez Segura M, Socarrás Ferrer BB, Macías Abraham C, del Valle Pérez LO, et al. Relevancia biológica y clínica del inmunofenotipaje celular en la leucemia linfoide aguda del niño. Rev Cuba Hematol Inmunol Hemoter. 2008 Apr;24(1):0-0. Disponible en: http://scielo.sld.cu/scielo.php?script=sci_arttext\&pid=S086 4-02892008000100006.

12. Kansagra A, Dahiya S, Litzow M. Continuing challenges and current issues in acute lymphoblastic leukemia. Leuk Lymphoma. 2018;59(3):526-41. DOI: 10.1080/10428194.2017.1335397.
13. PDQ Pediatric Treatment Editorial Board. Childhood Acute Lymphoblastic Leukemia Treatment (PDQ®): Health Professional Version. En: PDQ Cancer Information Summaries [Internet]. Bethesda (MD): National Cancer Institute (US); 2002 [citado 28 de julio de 2019]. Disponible en: http://www.ncbi.nlm.nih.gov/books/NBK65763/.

14. Dores GM, Devesa SS, Curtis RE, Linet MS, Morton LM. Acute leukemia incidence and patient survival among children and adults in the United States, 2001-2007. Blood. 2012 Jan 5;119(1):34-43. DOI: 10.1182/blood-2011-04-347872.

15. Rendón-Macías ME, Reyes-Zepeda NC, Villasís-Keever MÁ, Serrano Meneses J, Escamilla Núñez A. Tendencia mundial de la supervivencia en pacientes pediátricos con leucemia linfoblástica aguda: Revisión de las últimas cuatro décadas. Bol Méd Hosp Infant México. 2012 Jun;69(3):153-63. Disponible en: http://www.scielo.org.mx/scielo.php?script=sci_arttext\&pi $\mathrm{d}=\mathrm{S} 1665-11462012000300002$.

16. Hosmer DW, Hosmer T, Le Cessie S, Lemeshow S. A comparison of goodness-of-fit tests for the logistic regression model. Stat Med. 1997 May 15;16(9):965-80. DOI: $\quad$ https://doi.org/10.1002/(SICI)10970258(19970515)16:9<965::AID-SIM509>3.0.CO;2-0.

17. Gupta S, Howard SC, Hunger SP, Antillon FG, Metzger ML, Israels $\mathrm{T}$, et al. Treating Childhood Cancer in Low- and Middle-Income Countries. En: Gelband $H$, Jha $P$, Sankaranarayanan R, Horton S, editores. Cancer: Disease Control Priorities, Third Edition (Volume 3) [Internet]. Washington (DC): The International Bank for Reconstruction and Development / The World Bank; 2015 [citado 1 de enero de 2020]. Disponible en: http://www.ncbi.nlm.nih.gov/books/NBK343626/.

18. Rahman SA, Otim ME, Almarzouqi A, Rahman S. Setting Priorities in Childhood Cancer in Low Income Countries Using Nominal Group Technique: Experience from an International Childhood Cancer Forum Exercise in Bangladesh. Asian Pac J Cancer Prev APJCP. 2019 Jan 25;20(1):97-103. DOI: 10.31557/APJCP.2019.20.1.97.

19. Chow EJ, Leger KJ, Bhatt NS, Mulrooney DA, Ross CJ, Aggarwal S, et al. Paediatric cardio-oncology: epidemiology, screening, prevention, and treatment. Cardiovasc Res. 2019 Apr 15;115(5):922-34. DOI: 10.1093/cvr/cvz031.

20. Ezzat S, Rashed WM, Salem S, Dorak MT, El-Daly M, AbdelHamid M, et al. Environmental, maternal, and reproductive risk factors for childhood acute lymphoblastic leukemia in Egypt: a case-control study. BMC Cancer [Internet]. 2016 Aug 20 [citado 21 de enero de 2019];16. Disponible en: https://www.ncbi.nlm.nih.gov/pmc/articles/PMC4992254 /.

21. Steliarova-Foucher E, Colombet M, Ries LAG, Moreno F, Dolya A, Bray F, et al. International incidence of childhood cancer, 2001-10: a population-based registry study. Lancet Oncol. 2017 Jun;18(6):719-31. DOI: 10.1016/S14702045(17)30186-9.

22. Bona K, Blonquist TM, Neuberg DS, Silverman LB, Wolfe J. Impact of Socioeconomic Status on Timing of Relapse and Overall Survival for Children Treated on Dana-Farber Cancer Institute ALL Consortium Protocols (2000-2010). Pediatr Blood Cancer. 2016 Jun;63(6):1012-8. DOI: 10.1002/pbc.25928.

23. Gupta S, Sutradhar R, Guttmann A, Sung L, Pole JD. Socioeconomic status and event free survival in pediatric acute lymphoblastic leukemia: a population-based cohort 
study. Leuk Res. 2014 Dec;38(12):1407-12. DOI: 10.1016/j.leukres.2014.08.017.

24. Erdmann F, Kaatsch P, Zeeb H, Roman E, Lightfoot T, Schüz J. Survival from childhood acute lymphoblastic leukaemia in West Germany: does socio-demographic background matter? Eur J Cancer Oxf Engl 1990. 2014 May;50(7):134553. DOI: 10.1016/j.ejca.2014.01.028.

25. Terwilliger T, Abdul-Hay M. Acute lymphoblastic leukemia: a comprehensive review and 2017 update. Blood Cancer J. 2017 Jun 30;7(6):e577. DOI: 10.1038/bcj.2017.53.

26. Rytting ME, Jabbour EJ, O’Brien SM, Kantarjian HM. Acute lymphoblastic leukemia in adolescents and young adults. Cancer. 2017 Jul 1;123(13):2398-403. DOI: 10.1002/cncr.30624.

27. Schraw JM, Scheurer ME, Forman MR. A Vulnerable Age for the Introduction of Solid Foods in Pediatric Acute Lymphoblastic Leukemia. Nutr Cancer. 2017 Mar;69(2):261-6. DOI: 10.1080/01635581.2017.1263749.

28. Vizcaíno M, Lopera JE, Martínez L, Reyes ID los, Linares A. Guía de atención integral para la detección oportuna, diagnóstico, tratamiento y seguimiento de leucemia linfoide aguda en niños, niñas y adolescentes. Rev Colomb Cancerol. 2016 Jan 1;20(1):17-27. Disponible en: http://www.scielo.org.co/pdf/rcc/v20n1/v20n1a04.pdf.

29. Pui C-H, Evans WE. Treatment of acute lymphoblastic leukemia. N Engl J Med. 2006 Jan 12;354(2):166-78. DOI: 10.1056/NEJMra052603. 